\title{
ASHERMAN'S SYNDROME FOLLOWING THERMAL ABLATION OF THE ENDOMETRIUM
}

Sheila K. Pillai ${ }^{1}$, Bhuvana S², Jaya Vijayaraghavan ${ }^{3}$

\section{HOW TO CITE THIS ARTICLE:}

Sheila K. Pillai, Bhuvana S, Jaya Vijayaraghavan. "Asherman's Syndrome following Thermal Ablation of the Endometrium". Journal of Evolution of Medical and Dental Sciences 2014; Vol. 3, Issue 09, March 3;

Page: 2187-2193, DOI: $10.14260 /$ jemds/2014/2125

\begin{abstract}
Intrauterine adhesions develop as a result of varying degrees of intrauterine trauma. The extent of intrauterine adhesion formation and the impact of the adhesions on the contour of the uterine cavity vary widely. Extensive intrauterine adhesions with amenorrhea and hematometra can develop following endometrial ablation. Thirty one year old parous lady with history of thermal endometrial ablation for menorrhagia presented with complaints of amenorrhea and pain abdomen for two years. Hormonal assays were normal. Transvaginal scan showed absent endometrial stripe with patchy fluid collections in cavity suggestive of adhesions. The findings were corroborated by sonohysterogram and MRI pelvis. Patient did not respond to gonadotrophins. In view of persistent symptoms she was counseled for adhesiolysis under hysteroscopic guidance. Patient opted for hysterectomy. An atrophic uterus was noted intraoperatively and cut section revealed obliterated cavity with adhesive bands and mucoid collections supporting the diagnosis which was later confirmed on histopathological evaluation Extensive intrauterine adhesions with amenorrhea and hematometra can develop following endometrial ablation. Hysteroscopic adhesiolysis with cervical dilatation is the treatment of choice, but hysterectomy can be beneficial in a small subset of patients.
\end{abstract}

INTRODUCTION: Amenorrhea due to intrauterine adhesions was defined by Asherman in 1948 and is often referred to as Asherman's syndrome ${ }^{1}$. This syndrome results from acquired scarring of the endometrial lining, which prevents the normal build-up and shedding of the endometrium, leading to hypomenorrhoea or even amenorrhea. We are presenting a rare case of Asherman's syndrome which developed following non resectoscopic thermal ablation of the endometrium.

CASE REPORT: Mrs. X 31 year old multiparous lady (para 2, live 2 with previous two LSCS with tubal sterilization done) presented with complaints of amenorrhea and lower abdominal pain for the last two years. She had history of menorrhagia three years back and underwent thermal ablation of endometrium two years back in an outside hospital. She had been amenorrhoeic from then on and started to have lower abdominal pain which was cyclical initially and later progressed to continuous dull aching pain. She had two caesarean sections done ten years and four years back respectively. General examination was unremarkable. Abdomen was soft and cervix appeared healthy. Bimanual pelvic examination showed retroverted normal sized uterus with tenderness in adnexae. Baseline investigations were normal (hemogram, thyroid profile and serum prolactin). Serum FSH was 7.04 $\mathrm{mIU} / \mathrm{ml}$; Serum $\mathrm{LH}$ was $11.95 \mathrm{mIU} / \mathrm{ml}$ and serum estradiol was $44.59 \mathrm{pg} / \mathrm{ml}$. Transvaginal scan of pelvis showed uterus of size $7.7 \mathrm{~cm}$ x3.8 $\mathrm{cm}$. Endometrial stripe could not be visualized, with patchy collections of fluid measuring $8 \times 4 \mathrm{~mm}$ and $8 \times 3 \mathrm{~mm}$ in the cavity. Both ovaries were normal (Fig. 1). 


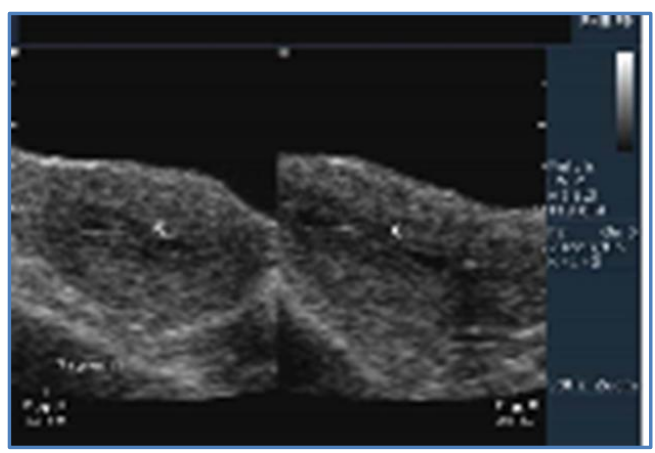

Fig. 1: Transvaginal scan pelvis showing patchy collections of fluid in the endometrial cavity with absent endometrial stripe

MRI scan of pelvis showed features suggestive of adenomyosis with bulky cervix and a band in the endometrium suggestive of possible adhesion. (Fig. 2).

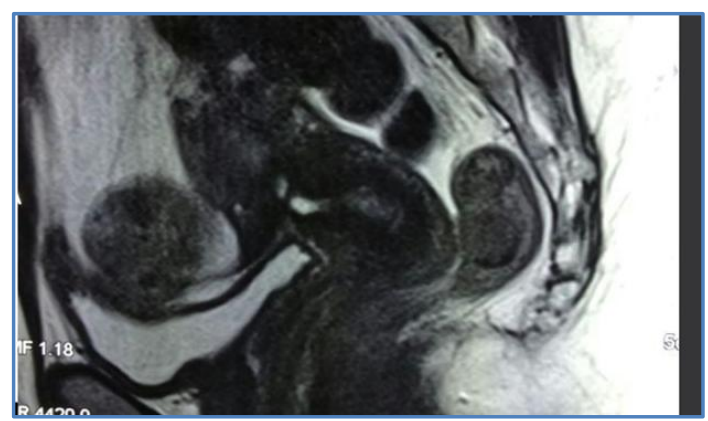

\section{Fig. 2: MRI pelvis showing bulky cervix with band} in the endometrium suggestive of adhesion

She was given a course of broad spectrum intravenous antibiotics and was planned for injection leuprolide acetate $3.75 \mathrm{mg}$ monthly IM for three doses. She reported back after two months with persistence of symptoms. Saline sonohysterogram was done which showed ballooned out cervix with obliterated uterine cavity suggestive of dense adhesions (Fig. $3 \& 4$ ).

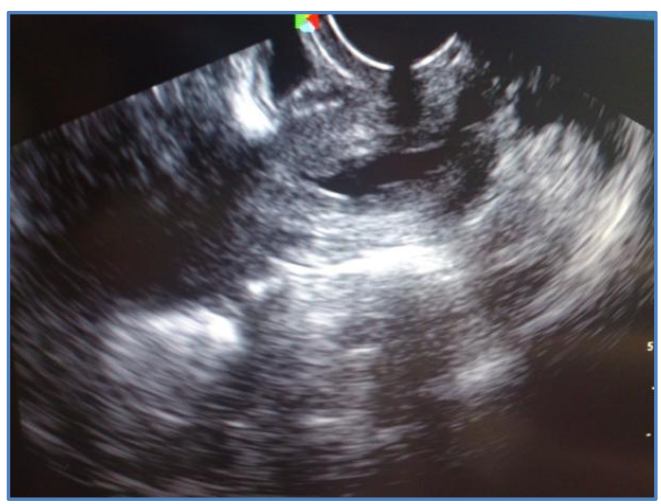

Fig. 3: Sonohysterogram showing ballooning of cervix 


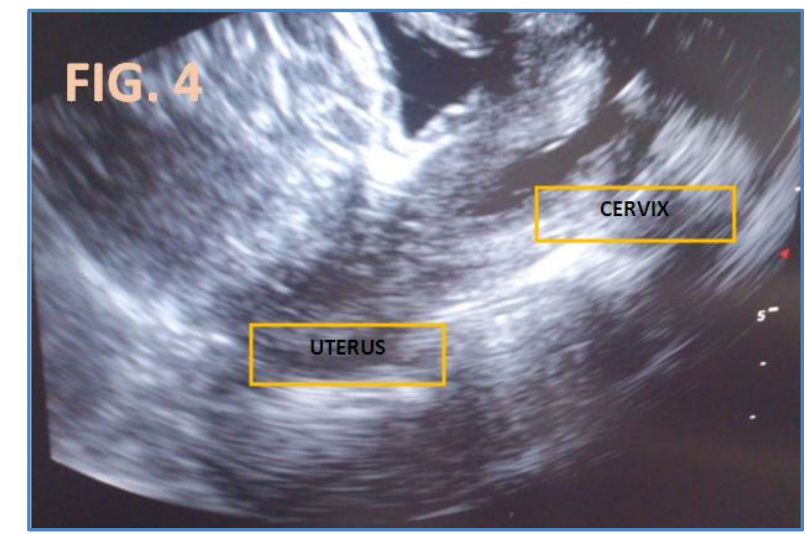

\section{Fig. 4: sonohysterogram showing obliterated endometrial cavity with ballooned out cervix}

She was effectively counseled about the treatment of adhesiolysis with hysteroscopic guidance. She was also counseled about the probable need for hysterectomy at a later date in the event of failure of this intervention especially in view of the dense adhesions. Patient opted for total abdominal hysterectomy as she had completed the family and wanted complete cure of symptoms permanently. Total abdominal hysterectomy with bilateral salpingectomy was done. Peroperative findings were atrophic uterus and normal ovaries (Fig. 5).

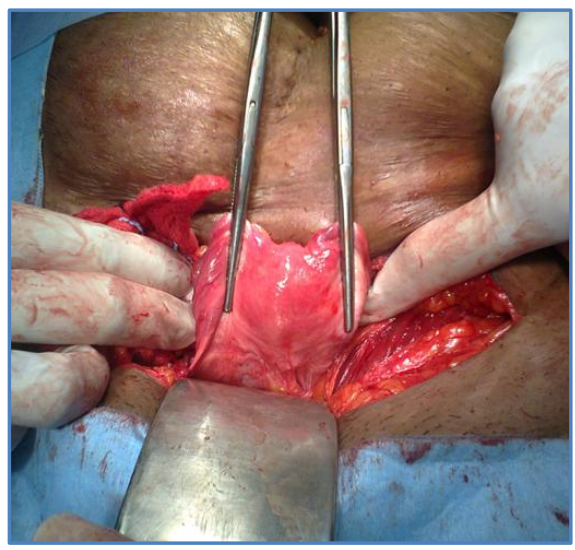

\section{Fig. 5: Intraoperative finding of atrophic uterus}

Cut section of the uterus showed obliterated endometrial cavity with adhesive bands and spaces filled with mucoid collections supporting the diagnosis (Fig. 6). 


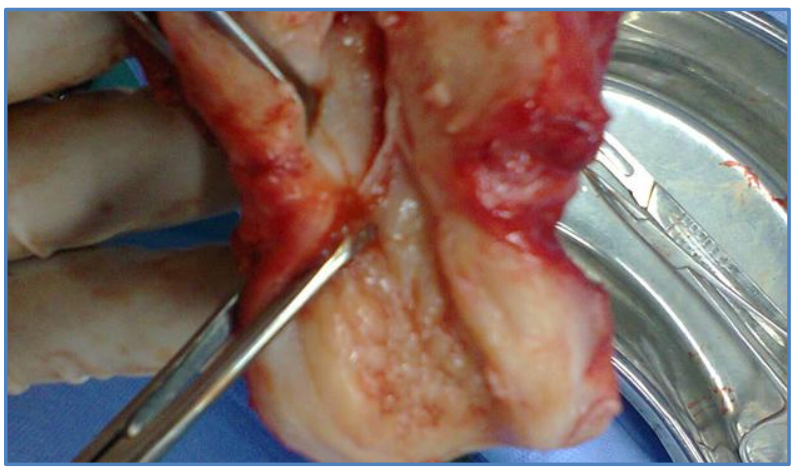

Fig. 6: cut section of uterus showing bands of adhesion

Histopathology report of uterus showed basal endometrium with normal myometrium, with cervix showing chronic papillary endocervicitis with squamous metaplasia.

DISCUSSION: Intrauterine adhesions develop as a result of varying degrees of intrauterine trauma. The extent of adhesion formation and the impact of the adhesions on the contour of the uterine cavity vary widely. With minimal disease, thin bands of tissue stretch between surfaces of the uterine cavity, whereas severe disease is characterized by complete obliteration of the cavity, with the anterior and posteriors wall of the uterus densely adherent. Majority of cases of severe intrauterine adhesive disease are related to curettage for pregnancy complications. Adhesions can develop in the nongravid uterus as a result of endometrial injury due to procedures such as myomectomy or nonpuerperal curettage 2 .

Endometrial ablation for women with abnormal uterine bleeding can be accomplished under hysteroscopic visualization using resectoscopic instruments, or can be performed with a nonresectoscopic ablation device, which when inserted into the uterine cavity delivers energy to uniformly destroy the endometrium. The endometrium must be destroyed or resected to the level of the basalis, which is approximately 4 to $6 \mathrm{~mm}$ deep, depending upon the stage of the menstrual cycle. Current non-resectoscopic technologies include bipolar radiofrequency (Novasure), hot liquid filled balloon (Therma Choice), cryotherapy (Her Option), circulating hot water (Hydro Therm Ablator), and microwave (Microwave Endometrial Ablation).

Therma Choice, Cavaterm and Thermablate EAS are the three available devices for thermal ablation $^{3}$. In women who underwent hot liquid filled balloon ablation with either Therma Choice, Cavaterm, or an unspecified device and were followed for four or more years, there was reduced uterine bleeding in 22 to 81 percent and amenorrhea in 23 to 58 percent ${ }^{4-6}$. Repeat ablation was performed in 5 to 11 percent, and hysterectomy in 2 to 13 percent.

The most common complications associated with endometrial ablation are uterine perforation, hemorrhage, hematometra and pelvic infection. Intrauterine scarring occurs following endometrial ablation. When areas of endometrium are adherent and there is endometrial bleeding behind the occlusion, hematometra will occur. The reported rate of hematometra in nonresectoscopic ablation is 0.9 percent and 2.4 percent in resectoscopic ablation 7 . This was supported by a study that found an association between postablation pain and a finding of hematometra in 19 women who underwent subsequent hysterectomy8. During endometrial ablation, uterine cavity 
occlusion is likely to occur in the cervical canal or at the lower uterine segment and hence ablation of the cervix or cervico-uterine junction should be avoided.

Intrauterine adhesions can be often asymptomatic with no clinical significance. Symptoms associated with significant intrauterine adhesions include infertility, menstrual irregularities (hypomenorrhea, amenorrhea) and cyclic pelvic pain. The extent of adhesions seen on hysteroscopy does not correlate well with the degree of menstrual irregularity and nearly 40 percent of patients with adhesions documented on hysteroscopy had no menstrual irregularities ${ }^{9}$ Hematometra should be suspected in a patient with history of endometrial ablation who presents with amenorrhea and cyclic pelvic pain, even remote from the procedure. Central hematometra is most likely to occur when the cervical canal is damaged at the time of endometrial ablation and has an incidence of 1 to 3 percent. ${ }^{10}$

The diagnosis of Asherman's syndrome is suggested by the absence of a normal endometrial stripe on pelvic ultrasound 11 , and may be further supported by the absence of withdrawal bleeding to administration of estrogen and then progestin for several weeks. The gold standard for the diagnosis of intrauterine adhesions is diagnostic hysteroscopy. Sonohysterogram, hysterosalpingogram (HSG) and transvaginal ultrasonography (TVUS) are other diagnostic imaging tools. After diagnosis, TVUS may be useful in predicting the outcome of surgical treatment of intrauterine adhesions. A study that evaluated seven women with severe adhesive disease prior to hysteroscopic resection found that women with a well-developed endometrial stripe resumed normal menses postoperatively, whereas women with minimal endometrial lining identified by ultrasound prior to surgery had an obliterated cavity, and did not respond to surgical intervention.12 Hematometra can be diagnosed by the visualization of pockets of echogenic fluid in the uterine cavity on transvaginal ultrasound. Central hematometra can usually be seen on imaging (ultrasound or MRI) at the time of cyclic pelvic pain.

Adhesions can arise from the endometrium, myometrium, or connective tissue. They vary in size from thin and fragile to thick and dense, with the ends broader than the middle. They may occur at the margins of the endometrial cavity or diffusely and in severe cases they completely obliterate the cavity. Marginal adhesions may appear crescent shaped or like a curtain. Mucosal adhesions are of the same color as the endometrium and fragile, whereas fibrous adhesions are pale and strong. Intrauterine adhesions due to tuberculosis tend to give the endometrial cavity a honeycomb appearance.

Various classification systems for intrauterine adhesions have been proposed. The system of the American Society of Reproductive Medicine consists of three stages of disease, based upon the extent of cavity involvement $(<1 / 3,1 / 3-2 / 3,>2 / 3)$, the type of adhesion seen at the time of hysteroscopy (filmy, filmy and dense, dense), and the patient's menstrual pattern (normal, hypomenorrhea, amenorrhea) 13.

The standard treatment of intrauterine adhesions is lysis under direct visualization using operative hysteroscopy. The goal of surgery is to restore the size and shape of the uterine cavity, as well as endometrial function. Care must be taken during cervical dilation in women with severe occlusion of the uterine cavity because it is easy to create false passages and perforate the uterus. Concurrent ultrasonography can help define the junction between the internal os and the intrauterine cavity, and guide dissection. Adhesions in the mid- to upper uterine cavity can be lysed under hysteroscopic guidance. Central hematometra can be treated by cervical dilatation. For persistent pain caused by hematometra despite minimally invasive treatment, hysterectomy may be 
indicated. Prevention of adhesion reformation can be achieved by administering high-dose estrogen therapy postoperatively, by placement of a bladder catheter (size 8 pediatric with a $5 \mathrm{cc}$ balloon) or intrauterine device (IUCD) into the uterine cavity immediately after lysis of adhesions. The bladder catheter is removed from the uterus after 10 days and the IUCD is removed after three months ${ }^{14}$. HSG or sonohysterogram can be performed two to three months after hysteroscopic resection of intrauterine adhesions for risk assessment for adhesion reformation.

The clinical presentation of amenorrhea with cyclic pelvic pain in a woman with history of endometrial ablation should always arouse the suspicion of intra uterine adhesions with probable hematometra and cervical stenosis. The diagnosis should be confirmed by combining imaging modalities with hormonal assays. A challenge test with progestin alone and if negative with estrogen - progestin combination is mandatory. The gold standard for treatment is hysteroscopic adhesiolysis combined with cervical dilatation if warranted. Adequate counseling should be given about the reduced success rates of this treatment modality in cases where the adhesions are severe with near total obliteration of the endometrial cavity. If adhesiolysis fails, with persistence or subsequent recurrence of clinical symptoms, hysterectomy will be the preferred choice especially in those who have completed their families.

In the case we have reported, the patient had already completed her family. She was diagnosed to have severe intrauterine adhesions on evaluation. She was appropriately counseled about the primary option of lysis of adhesions with hysteroscopic guidance. She opted for hysterectomy as the modality of treatment to achieve total and permanent relief of symptoms.

SUMMARY: Extensive intrauterine adhesions with amenorrhea and hematometra can develop following endometrial ablation. Hysteroscopic adhesiolysis with cervical dilatation is the definitive treatment of choice, but hysterectomy can be beneficial in a small subset of patients.

\section{REFERENCES:}

1. Asherman JG. Traumatic intrauterine adhesions. J Obstet Gynaecol Br Emp 1950; 57:892.

2. Al-Inany H. Intrauterine adhesions. An update. Acta Obstet Gynecol Scand 2001; 80:986.

3. www.ethicon360.com/products/gynecare-thermachoice-uterine-balloon-therapy-system

4. Amso NN, Fernandez H, Vilos G, etal. Uterine endometrial thermal balloon therapy for the treatment of menorrhagia: long-term multicenter follow-up study. Hum Reprod 2003; 18:1082.

5. Ahonkallio S, Martikainen H, Santala M. Endometrial thermal balloon ablation has a beneficial long-term effect on menorrhagia. Acta Obstet Gynecol Scand 2002; 87:107.

6. Meetler L. Long-term results in the treatment of menorrhagia and hypermenorrhea with a thermaballoon endometrial ablation technique. JSLS 2002; 6:305.

7. Lethaby A. Hickey M, Garry R, Pennix J. Endometrial resection /Ablation techniques for heavy menstrual bleeding. Cochrane Database Syst Rev 2009; CD001501.

8. Carey ET, El-Nashar SA, Hopkins MR et al. Pathologic characteristics of hysterectomy specimens in women undergoing hysterectomy after global endometrial ablation. J Minim Invasive Gynecol 2011; $18: 96$.

9. Letterie GS, Structural abnormalities and reproductive failure: effective techniques for diagnosis and management, Blackwell Science, Massachusetts 1998. 


\section{CASE REPORT}

10. McCausland AM, McCausland VM. Long-term complications of endometrial ablation: cause, diagnosis, treatment and prevention. J Minim Invasive Gynecol 2007; 14:399.

11. Hubert SR, Marcus PS, Rothenberg JM etal. Hematometra after thermal balloon endometrial ablation in a patient with cervical incompetence. J Laparoendose Adv Surg Tech A 2001; 11:311.

12. Schlaff WD, Hurst BS. Preoperative sonographic measurement of endometrial pattern predicts outcome of surgical repair in patients with severe Asherman's syndrome. Fertil Steril 1995; 63:410.

13. The American Fertility Society classifications of adnexal adhesions, distal tubal occlusion, tubal occlusion secondary to tubal ligation, tubal pregnancies, mullerian anomalies and intrauterine adhesions. Fertil Steril 1988; 49:944.

14. Orhue AA, Aziken ME, Igbefoh JO. A comparison of two adjunctive treatments for intrauterine adhesions following lysis. Int J Gynaecol Obstet 2003; 82: 49.

\section{AUTHORS:}

1. Sheila K. Pillai,

2. Bhuvana $\mathrm{S}$,

3. Jaya Vijayaraghavan

\section{PARTICULARS OF CONTRIBUTORS:}

1. Assistant Professor, Department of Obstetrics and Gynaecology, Sri Ramachandra Medical College and Research Institute, Porur, Chennai.

2. Associate Professor, Department of Obstetrics and Gynaecology, Sri Ramachandra Medical College and Research Institute, Porur, Chennai.
3. Professor, Department of Obstetrics and Gynaecology, Sri Ramachandra Medical College and Research Institute, Porur, Chennai.

\section{NAME ADDRESS EMAIL ID OF THE} CORRESPONDING AUTHOR:

Dr. Sheila K. Pillai, Assistant Professor, Sri Ramachandra Medical College and Research Institute, Porur, Chennai - 600116.

E-mail: drsheilasunil@hotmail.com

Date of Submission: $11 / 02 / 2014$.

Date of Peer Review: 12/02/2014.

Date of Acceptance: 18/02/2014.

Date of Publishing: 26/02/2014. 